\title{
SOME OBSERVATIONS ON ANOREXIA NERVOSA
}

\author{
Cecil B. KidD, M.D., Ph.D., D.P.M. \\ J. F. Wood, M.B., D.P.M. \\ Senior Lecturer \\ Clinical Tutor
}

Department of Mental Health, University of Aberdeen

\begin{abstract}
DISTURBANCES of gastro-intestinal function and of nutrition are to be found as a cause of, a correlate with or a consequence to almost every form of psychiatric illness (Millar, 1953). Among these, the distinct entity of anorexia nervosa has attracted much attention from psychiatrists, general physicians, endocrinologists and biochemists. Although anorexia nervosa is a rare condition, the clinical interest accorded to it reflects both the severity of its impact on the patient and the major therapeutic challenge it presents to the clinician. The disease process comprises a complex interplay of physical, psychological, endocrinological, metabolic and electrolytic dysfunction; treatment is very lengthy, relapse is frequent and a fatal outcome is not unknown.
\end{abstract}

The diagnosis of anorexia nervosa rests on the recognition of a triad of symptoms which are universally accepted as pathognomonic of this condition; a gross loss of weight leading to emaciation, a failure to eat, and disturbance of menstrual function leading to oligomenorrhoea and amenorrhoea. The psychological concomitants of this state also have been described as a triad; denial of hunger despite prolonged inanition, denial of thinness despite extreme emaciation, and denial of fatigue despite excessive and frantic activity done in a state of chronic underfeeding (Mayer, 1963). A comprehensive clinical description of this disorder was provided by Sir William Gull who coined the term "anorexia nervosa" in 1874. He observed its occurrence in young women who presented with "great emaciation, amenorrhoea, constipation, anorexia alternating occasionally with a voracious appetite, restlessness, activity, peevishness of temper and a feeling of jealousy, together with an absence of any organic cause". He ascribed this to a morbid mental process, he commented that the patients' activity and sense of well-being were grossly out of proportion to their inanition, and observed shrewdly that the family were generally the worst attendants (Gull, 1874). The early detection of anorexia nervosa is vital to offsetting the hazard it presents to the health and life of these patients and, as Stafford-Clark (1958) has rightly pointed out, the management required both uniquely illustrates and emphasizes the necessity to resolve any dichotomy between physiological and psychological approaches in treatment.

\section{Prevalence and Surveys}

Anorexia nervosa is a rare condition, yet not so rare that several new cases will present to clinicians each year at every major centre of population. Study of patient records at Aberdeen covering two selected periods 1949-1956 and 1960-1965 inclusive revealed a firm diagnosis of anorexia nervosa in 18 and 12 patients respectively who had been treated in the Professorial Psychiatric Unit. In a study in the North-east region of Scotland of all persons over the age of 15 years newly referred to a psychiatrist during one year, three of the 1,240 new women patients thus identified had a diagnosis of anurexia nervosa (Innes and Sharp, 1962). Despite the paucity of cases, meaningful numbers for study and follow-up have been accumulated in several on-going and retrospective enquiries. Among these, Bruch (1962) studied in detail the psychological characteristics of conceptual disturbances and disorders of perception in nine women admitted for treatment of anorexia nervosa. Crisp (1965a) studied the clinical patterns and outcome of treatment in 27 women with anorexia nervosa and in another enquiry (Crisp, 1965b) presented data on the evolution of this condition and the patient characteristics of a series of 42 affected women. Dally and Sargant (1960) carried out a treatment study comparing results from the then new and now widely used regime of chlorpromazine combined with modified insulin therapy in 20 patients with other treatment regimes previously employed in a series of 24 patients. Hawkings, Jones, Sim and Tibbetts (1956) reported a number of patients with "deliberate disability" and drew interesting comparisons between this group and five patients 
who were treated in the same unit for anorexia nervosa. In the same paper the authors described the results of a postal follow-up enquiry on 23 patients treated for anorexia nervosa during the ten year period 1938 to 1948 . Kay and Leigh (1954) reported on a definitive series of 34 women who were treated at the Maudsley Hospital for anorexia nervosa between 1932 and 1952 and described in detail their clinical features and outcome. King (1963) studied a series of 21 anorexic patients to delineate from anorexia nervosa those whose anorexia was secondary to other psychiatric illness, and to identify the significant background factors and clinical characteristics of the former. Another major survey was reported by Nemiah (1950) who selected 14 patients on the basis of the classical symptom triad and in whom no evidence of gross disease had been found in a primarily aetiological role: as well as reporting clinical observations this study detailed findings from enquiry into and measurement of the patients' personality profiles, attitudes and interpersonal relationships. Patient groups were accumulated for research on the metabolic and endocrinological aspects of anorexia nervosa and results have been reported recently: delta glucose values before and after a high calorie high carbohydrate dietary regime were studied in nine patients, the regulation of water balance was studied in 12 patients (Russell, 1965), and gonadotrophin and oestrogen excretion was studied in seven severely emaciated women (Russell, Loraine, Bell and Harkness, 1965). Seventeen patients with anorexia nervosa and who showed hypokalaemia on laboratory investigation were studied by Wigley (1960) who identified and commented on the high occurrence of renal disorder among this group. The clinical state, management and progress of 53 patients with anorexia nervosa who were treated at the London Hospital between 1897 and 1957, the largest series to have been published, was reported by Williams (1958); this paper emphasizes especially the relationships between treatment procedures and outcome.

In addition to these major surveys of patients with anorexia nervosa considerable clinical, psychological and biochemical data have accrued from smaller series and individual case reports which further advance medical understanding of this condition.

\section{Patient Characteristics}

All patients who have anorexia nervosa are females since amenorrhoea is widely regarded as essential to the accurate diagnosis of this condition. Some authors have included males in this diagnostic category (Bruch, 1962; Dally and Sargant, 1960; Kay and Leigh, 1954; Russell and others, 1965) but, as Dally and Sargant (1960) have pointed out, the occurrence of convincing anorexia nervosa-like states in men is most uncommon. Anorexia nervosa characteristically occurs among adolescent or young women (Williams, 1958). In Crisp's (1965b) series of 27 women, all but two were adolescents with a mean age of 17 years. In his study of 40 patients, it was found that all but seven had an onset of illness before age 21 (Crisp, 1965a). Similarly Nemiah (1950) found the age at onset of illness to be between 14 and 24 years (mean age 18 years) in his series and Kay and Leigh (1954) showed that 70 per cent of their patients had their first symptoms before reaching the age of 26 years. The age patterns on admission to the Professorial Psychiatric Unit in Aberdeen of 12 patients studied from 1960 to 1965 showed that half were below 25 years, a proportion identical to the similar data from the Guy's Hospital series (StaffordClark, 1958), but as Loeb (1964) has observed, many post-adolescent patients will have had episodes of anorexia in adolescence, although perhaps not manifesting them to such a degre as to have called for medical intervention dis the time. Crisp (1965a) showed that the typicat patient had a higher birth-weight than her sisters, was much more likely to have been bottle-fed in infancy, or if breast-fed to have had prolonged breast feeding, to have been well-nourished or over-nourished in childhood and to have had an early menarche. These characteristics were found not to have been influenced by the factors of race, social class maternal age and parity, length of gestation or genetic inheritance. Most patients are described as being "plump" before the onset of illness. Often they are awkward, reserved, and physically unattractive (Wall, 1964). The association between the onset of illness and a crash diet programme to reduce weight occurs too frequently to be a fortuitous occurrence (Crisp, 1965a; Mayer, 1963; Nemiah, 1950). In contrast to the insightless extremes of self-deprivation to which these patients subject themselves, it is a characteristic common to the majority that they are talented and of high intelligence (King, 1963; Wall, 1964).

\section{Physical Features}

The physical appearance of the patient is marked by obvious emaciation. The facies suggest that the patient is older than her stated 
age, skeletal structure is clearly defined and pallor is invariably present. The patient has an intolerance to cold. Fatigue and apathy while obvious are forcefully denied. Atrophy of the breasts and of buttock fat are common. Where the latter is marked the sagging folds of skin take on the classical appearance of "elephant skin". In early phases of weight reduction loss of fatty tissue predominates. Extreme weight loss in later emaciated patients indicates rapid katabolism of non-fatty tissue reserves. Hair distribution is normal, but may be augmented by facial and limb hirsuties and a fine lanugolike downy growth of hair on the body. Amenorrhoea is invariably present. In similar proportions of patients, menstrual disturbance and anorexia appear together as the first features of the condition, menstrual disturbance precedes all other physical features, or follows during the course of established weight reduction (King, 1963). On examination, bradycardia, hypotension, diminished sweating and oedema are commonly found. Constipation is always present. Abdominal tenderness may be elicited. Gynaecological examination normally reveals an infantile uterus and atrophic changes in the vaginal mucosa due to depressed oestrogen function. Restlessness and excessive activity are normally observed, always to a degree out of proportion to the clinician's assessment of the emaciated patient's physical reserves.

\section{Metabolic Features}

The basal metabolic rate is low. The excretion of 17-ketosteroids and oestrogenic substances is also markedly reduced. The Kepler test for hypoadrenal-cortical function is frequently positive (Wall, 1964). Crisp (1965a) has demonstrated recently that while patients with anorexia nervosa have a flat glucosetolerance curve due to sustained high blood glucose levels which is reversible with weight gain, high plasma insulin levels persist. Paradoxically, this pattern is usual in obese subjects. It is not known whether this is present before the onset of illness. A further metabolic likeness of the anorexic to the obese patient was demonstrated by analysis of the content in weight gain among patients with anorexia nervosa under treatment: disproportionately high fat deposits were found, a similar pattern to that expected among over-feeding normal persons (Russell and Mezey, 1962). Russell (1965) and Russell and others (1965) carried out three studies aimed at testing the hypothesis that anorexia nervosa is associated with hypothala- mic dysfunction. In the first, delta glucose values shown by glucose tolerance tests were found to be high but reversible when the patients were fed on a high-carbohydrate highcalorie dietary intake. Russell has concluded from this that the metabolic change is secondary to chronic carbohydrate deprivation and can be corrected by adjusting the diet. The second study, of regulation of water balance in these patients, sought to explain the findings that many patients with anorexia nervosa are oedematous and have a reduced capacity to excrete a water load, yet urinary concentration remains normal. It was found that ability to excrete a water load depended not on weight loss but on duration of illness. Antidiuretic hormone assay revealed normal levels, glomerular filtration rate showed some reduction and it was again concluded that this metabolic disturbance is the result of malnutrition, is correctable over time, and it is not due to a primary hypothalamic dysfunction. The third study sought to gain data on hormone levels and on the effect of malnutrition on endocrine function in anorexia nervosa patients by examining the excretion of human pituitary gonadotrophins and oestrogens before and after treatment. It was found that before treatment the output of these hormones was reduced. There was also disproportionate decrease in oestriol compared with decreases in oestradiol and oestrone. After feeding the oestrogen excretion returned to a normal pattern and quantity, indicating a secondary process (the disproportionate reduction in oestriol could be due to the way in which oestrogens are metabolised in malnutrition), but the gonadotrophin levels did not rise to the extent that normal cyclical activity was resumed. It was concluded that while many metabolic effects of anorexia nervosa are secondary to malnutrition, the existence of a primary hypothalamic defect might conceivably account for some of the endocrine changes met in this condition.

Electrolyte deficiency may occur in anorexia nervosa. Sunderman and Rose (1948) first described the occurrence of hypokalaemic alkalosis and Wigley (1960) reported and reviewed 17 cases of anorexia nervosa all of whom had hypokalaemia, some of whom had hyponatraemia and eight of whom showed evidence of renal dysfunction. This study underlines the importance of recognising potassium depletion in anorexia nervosa which may lead to renal complications such as tubular vacuolation. As well as this, the patient with anorexia nervosa takes 
very little fluids, urinary output is low, many have a negative nitrogen balance and a tendency to osteoporosis, and the combination of oliguria with hypercalcaemia may further impair renal efficiency by calculus formation (Wall, 1964).

\section{Psychological Features}

Anorexia nervosa is primarily a psychogenic disorder which leads to and is complicated by physiological and pathological events. The prime mover in this debilitating condition is, however, the psychogenic factor (Wall, 1964). Consistent patterns of pre-morbid personality attributes, social attitudes and maladjustments, and behavioural anomalies during the course of the illness all testify to the presence in anorexia nervosa of a gross psychiatric disorder. Normally it is found that the patient before becoming ill is described as stubborn, strongminded, determined, of high ideals, not amenable to reason, self-willed and overly sensitive (Palmer and Jones, 1939). Wall (1964) has stressed the distinctly introverted personality of the patient, her meticulousness and obsessionality and an intellectual superiority which is at variance with an attitude of unmitigated stubborness. Lack of humour and irritability which were shown by King (1963) to be personality facets of the pre-anorexic patient concord well with Gull's original observation of "peevishness" (Gull, 1874). An abnormal degree of dependency by the patient on a parent, usually a characteristically dominant and restrictive mother, is very commonly seen (King, 1963). Many patients feel isolated and at best attain only a poor social adjustment (Nemiah, 1950). The quality of the patient's dependency on her mother has been studied by Crisp (1965a) who postulates a relationship between the typical patient's pre-anorexic plumpness and the mother's neurotic need to over-feed her. However, by no means all patients have been fat during adolescence and childhood. The onset of anorexia nervosa following a voluntary dieting regime occurs only in onethird to one-half of patients (Kay and Leigh, 1954; Nemiah, 1950) and Loeb (1964) has stressed the frequency of occurrence of other precipitating conflicts in anorexic patients which are unrelated to the drive to diet. All patients with anorexia nervosa do have, however, a pathological distortion of their own body image. While emaciation is not only obvious but extreme, the patient denies being thin and defends her appearance as being normal and right. This distortion is not amenable to reason (Bruch, 1962; Mayer, 1963).

A dominant psychological feature common to all patients is their preoccupation with and disgust for sexual thought and development. Attitudes of revulsion and frigidity are either expressed or thinly veiled by protestations of alleged ignorance of sexual matters. The symbolic relationship between feeding and sexual pleasure is well known in psychological theory and many authors have illustrated from detailed psychological investigation of anorexic patients that unconscious fantasies of oral impregnation, repressed in early life, can be revived in the stress of puberty and may play a contributory role in the genesis of anorexia nervosa (Grimshaw, 1959; McCullagh and Tupper, 1940; Waller, Kaufman and Deutsch, 1940). Certainly the sexual histories of many patients contain an account of some sexual incident, often trivial but frightening to the patient, around the time of onset of anorexia nervosa (Crisp, 1965b), but although attitudes of conflict and hostility to sexuality, fears of pregnancy and poor heterosexual adaptation are invariable concomitants of the condition, factors other than purely a disorder in psychosexual adjustment are heldo generally to be essential to its development.

Denial of hunger is as striking a clinicako feature in these patients as is a denial of thin-? ness. Encouragement to eat is met with hostility and complaints of unbearable abdominal fullness follow the ingestion of even small amounts of food. The patient frequently complains of constipation, seeks for purgatives, or may induce vomiting to evacuate the stomach contents. She will go to enormous lengths to avoid taking food and will hide it or dispose of it unless arrangements are made to prevent this.

Denial of fatigue has also been emphasized by Mayer (1963) as a significant feature in the patient. Overactivity and restlessness are observed and the patient expresses a subjective feeling of alertness and a distaste for idleness. Despite her often intelligent awareness of the caloric cost of exercise, the patient will normally make every contrary effort to further jeopardise her limited energy reserves unless she is induced to do otherwise. The clinician is often struck by the patient's determined efforts to follow the path towards, seemingly, her self-destruction.

Understandably patients with anorexia nervosa are by their psychological characteristics at least ambivalent or at most hostile to therapeutic efforts which are aimed at their resusci- 
tation and recovery. Some patients revoke from treatment to the grave injury of their already precarious clinical state. Many develop emotional reactions of withdrawal, depression and anxiety in the face of the treatment regime and these secondary psychiatric manifestations require recognition and management in their own right.

\section{Treatment and Outcome}

Wall (1964) commented that the most important element in treatment of anorexia nervosa is the actual recognition of the illness as a psychiatric disorder, one which demands from the clinician all the sustained effort and enthusiasm required for the management of a patient with interacting physical and psychological dysfunction. Medical, endocrine, metabolic and psychiatric aspects of treatment cannot be considered separately. Mayer's examination of the literature revealed a variety of reportedly efficaceous but diverse treatment approaches. Hormonal methods have been employed (oestrogens, thyroid, pituitary extracts, ACTH and cortisone); forced feeding, intubation or intravenous fluid therapy have been reponted to have brought about remission; and psychotherapeutic methods without physical treatments have been found beneficial in some cases (Mayer, 1964). Williams (1958) stressed the primary importance of the nutritional aspects of treatment, basing this conclusion from his uniformly successful results in patients fed by intubation, and suggested that specialised psychotherapy was not indicated in treatment. In general, polemic viewpoints about approach to the treatment of anorexia nervosa are modified by the clinician's awareness of the indivisible interplay of organic and psychological factors which present in this condition. A critical formulation of the therapeutic priorities in each anorexic patient's clinical presentation is required.

The most successful treatment regime appears to be the joint use of insulin and chlorpromazine or reserpine, given in combination with detailed attention to the patient's nutritional requirements and psychological needs. This regime was described by Dally, Oppenheim and Sargant (1958) and by Davidson and Nabney (1959) and its superionity over other forms of treatment was evidenced first by Dally and Sargant in 1960. This treatment programme is followed at Aberdeen, as in many other centres in Britain and abroad, with enoouraging results. Each patient is confined to bed so that energy is conserved, observation is possible and food intake can be supervised. Chlorpromazine is prescribed in increasing dosage according to tolerance. Soluble insulin is given by intramuscular injection in increasing dosage and the resultant hypoglycaemia is terminated when signs of sweating and drowsiness appear. Anabolics, vitamins and high calorie food supplements are also given. Intubation is avoided as it is psychologically undesirable and rarely required. Throughout the early stages of treatment supportive psychotherapy is employed to provide encouragement, an atmosphere of understanding and to mold the basis of the doctor/patient therapeutic relationship. As the patient improves the drugs are reduced, graduated exercise is allowed and psychotherapy is employed to aid the patient in making the necessary readjustments in her psychological functioning that will both militate against relapse and favour better personality and social integration. Mayer (1963) has emphasized rightly that psychotherapy should be directed towards specific aspects of the patient's abnormal emotional characteristics. He has described this as a re-educative procedure whereby the patient must gain the insight to learn, first, "to see herself as others see her, as an abnormally and unaesthetically thin individual"; second, to feel hungry and to want to react to hunger by desiring food; and third, to feel fatigue as others do. In short, psychotherapy here aims to reverse the characteristic triad of denilals. In most cases, psychotherapeutic support or management is required for some time after the patient has fully regained normal nutritional and menstrual functioning.

Follow-up for at least one year is essential (Dally and Sargant, 1960). Studies of outcome reveal much about the natural history of anorexia nervosa and the efficacy of treatment. Relapse may occur and a fatal outcome although now rare is still occasionally seen. Of 30 patients treated in Aberdeen between 1949 and 1965 only one is known to have died, by suicide. Follow-up of William's (1958) series of 53 patients admitted to the London Hospital during a 60 year period showed that 10 had died, 3 had not improved, 33 had improved or recovered and 7 could not be traced. Hawkings and others (1956) traced 15 of their series of 23 patients, all of whom had recovered. Dally and Sargant (1960) found that of 20 patients treated with insulin and chlorpromazine, none died, only 3 relapsed and most made a good recovery to normal weight and normal menstruation. Stafford-Clark (1958) found on one year follow-up of 13 patients that 9 were either 
completely well or had some residual symptoms yet none sufficient to interfere with daily life, only one patient was still disabled by symptoms and three could not be traced. Kay and Leigh (1954) found on follow-up after periods from 2 to 19 years that about half of the Maudsley series of patients still had some menstrual abnormality, disturbance of appetite or fluctuating weight and eight patients had died, 3 from the effects of anorexia nervosa, the remainder from other causes. Crisp (1965a) studied the outcome of treatment in 21 patients two-anda-half years after discharge; 2 had died, 17 had regained normal weight of whom 12 exhibited normal eating behaviour, and 11 had established menstrual cycles. Crisp drew the meaningful conclusion that treatment based on refeeding alone is not enough and is as unlikely to be successful as unsupported attempts at superficial manipulation of the patient's life situation. Physical and psychological treatments both are required. In anorexia nervosa, where psychological and physiopathological factors combine in producing a severe and debilitating disorder, a successful outcome depends on an awareness of these two components and on a programme of patient management based on this recognition.

\section{REFERENCES}

Bruch, H. (1962): Perceptual and Conceptual Disturbances in Anorexia Nervosa, Psychosom. Med., 24, 187.

CrISP, A. H. (1965a): Some Aspects of the Evolution, Presentation and Follow-Up of Anorexia Nervosa, Proc. roy. Soc. Med., 58, 814.

Crisp, A. H. (1965b): Clinical and Therapeutic Aspects of Anorexia Nervosa: a Study of 30 Cases, J. psychosom. Res., 9, 67.

Dally, P. J., OPPENheim, G. B., and Sargant, W. (1958): Anorexia Nervosa, Brit. med. J., ii, 633.

$\checkmark$ Dally, P. J., and Sargant, W. (1960): A New Treatment of Ánorexia Nervosa, Brit. med. J., i, 1770.

Davidson, J. C., and NABNEY, J. B. (1959): A Case of Anorexia Nervosa Treated by a Combination of Psychotherapy, Insulin, and Reserpine, Ulster med. J., 28, 205.
Grimshaw, L. (1959): Anorexia Nervosa: a Contribution to its Psychogenesis, Brit. J. med. Psychol., 32, 44.

$\checkmark$ Gull, W. W. (1874): Anorexia Nervosa, Trans. Clin. Soc. Lond., 7, 22.

$\checkmark$ Hawkings, J. R., Jones, K. S., Sim, M., and Tibberts, R. W. (1956): Deliberate Disability, Brit. med. J., i, 361 .

INNES, G., and Sharp, G. A. (1962): A Study of Psychiatric Patients in North-easit Scotland, J. ment. Sci., 108, 447.

$\checkmark$ KaY, D. W. K., and LeIGH, D. (1954): The Natural History, Treatment and Prognosis of Anorexia Nervosa, J. ment. Sci., 100, 411.

KING, A. (1963): Primary and Secondary Anorexia Nervosa Syndromes, Brit. J. Psychiat., 109, 470.

Loeb, L. (1964): The Clinical Course of Anorexia Nervosa, Psychosomatics, 5, 345.

$\sqrt{ }$ MAYer, J. (1963) : Anorexia Nervosa, Postgrad. Med., 34, 529.

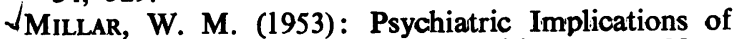
Disturbances of Eating and Nutrition, Proc. Nutr. Soc., 12, 144.

MoCullagh, E. P., and Tupper, W. R. (1940): Anorexia Nervosa, Ann. intern. Med., 14, 817.

Nemiah, J. C. (1950): Anorexia Nervosa. A Clinical Psyohiatric Study, Medicine (Baltimore), 29.225.

PALMER, H. D., and JoneS, M. S. (1939): Ánorexia Nervosa as a Manifestation of Compulsion Neurosis, Arch. Neurol. Psychiat. (Chic.), 41, 856.

/Russell, G. F. M. (1965): Metabolic Aspects of Anorexia Nervosa, Proc. roy. Soc. Med., 58, 811.

Russell, G. F. M., Loraine, J. A., Bell, E. T., and HARKNESS, R. A. (1965): Gonadotrophin and Oestrogen Excretion in Patients with Anorexiace Nervosa, J. Psychosom. Res., 9, 79.

Russell, G. F. M., and Mezey, A. G. (1962): An Analysis of Weight Gain in Patients with Anorexia Nervosa Treated with High Calorie Diets, Clin. Sci., 23, 449.

$\checkmark$ StAFFoRD-Clark, D. (1958): Anorexia Nervosa, Brit. med. J., ii, 446.

Sunderman, F. W., and Rose, E. (1948): Studies in Serum Eleotrolytes: Changes in the Serum and Body Fluids in Anorexia Nervosa, J. clin. Endocr., 8, 209.

Wall, N. M. (1964): Anorexia Nervosa, Psychosomatics, 5, 157.

Waller, J. V., Kaufman, M. R., and Deutsch, F. (1940): Anorexia Nervosa: a Psychosomatic Entity, Psychosom. Med., 2, 3.

Wigley, R. D. (1960): Potassium Deficiency in Anorexia Nervosa, with Reference to Renal Tubular Vacuolation, Brit. med. J., ii, 110.

'Williams, E. (1958): Anorexia Nervosa: a Somatic Disorder, Brit. med. J., ii, 190. 